\title{
Celecoxib Does Not Protect against Fibrosis and Inflammation in a Carbon Tetrachloride-Induced Model of Liver Injury ${ }^{\text {\} }$
}

\author{
Todd R. Harris, Sean Kodani, Amy A. Rand, Jun Yang, Denise M. Imai, Sung Hee Hwang, \\ and Bruce D. Hammock
}

Department of Entomology and Nematology and UC Davis Comprehensive Cancer Center (T.R.H., S.K., A.A.R., J.Y., S.H.H., B.D.H.), and Comparative Pathology Laboratory, School of Veterinary Medicine (D.M.I.), University of California, Davis, California

Received January 18, 2018; accepted May 18, 2018

\section{ABSTRACT}

The cyclooxygenase-2 (COX-2) selective inhibitor celecoxib is widely used in the treatment of pain and inflammation. Celecoxib has been explored as a possible treatment of liver fibrosis with contradictory results, depending on the model. The present study reports the effect of celecoxib in a 5-week carbon tetrachloride $\left(\mathrm{CCl}_{4}\right)$-induced liver fibrosis mouse model. Celecoxib alone and in combination with inhibitors of the enzymesoluble epoxide hydrolase (sEH), as well as a dual inhibitor that targets both COX-2 and $\mathrm{sEH}$, were administered via osmotic minipump to mice receiving intraperitoneal injections of $\mathrm{CCl}_{4}$. Collagen deposition was elevated in the mice treated with both celecoxib and $\mathrm{CCl}_{4}$ compared with the control or $\mathrm{CCl}_{4}$-only groups, as assessed by trichrome staining. Histopathology revealed more extensive fibrosis and cell death in the animals treated with both celecoxib and $\mathrm{CCl}_{4}$ compared with all other experimental groups. Although some markers of fibrosis, such as matrix metalloprotease, were unchanged or lowered in the animals treated with both celecoxib and $\mathrm{CCl}_{4}$, overall, hepatic fibrosis was more severe in this group. Cotreatment with celecoxib and an inhibitor of $\mathrm{sEH}$ or treatment with a dual inhibitor of COX-2 and SEH decreased the elevated levels of fibrotic markers observed in the group that received both celecoxib and $\mathrm{CCl}_{4}$. Oxylipid analysis revealed that celecoxib reduced the level of prostaglandin $\mathrm{E}_{2}$ relative to the $\mathrm{CCl}_{4}$ only group. Overall, celecoxib treatment did not decrease liver fibrosis in $\mathrm{CCl}_{4}$-treated mice.

\section{Introduction}

Celecoxib is a nonsteroidal anti-inflammatory drug (NSAID) used by millions of patients to alleviate the pain and inflammation associated with diseases such as rheumatoid arthritis (Bessone et al., 2016). Reviews of controlled trials have found no significant difference in the incidence of liver damage between patients administered celecoxib and those receiving placebo (Bessone et al., 2016).

Liver fibrosis is the result of a normally beneficial woundhealing process that can be initiated by toxicants such as

Primary laboratory of origin: Hammock.

This work was funded by the National Institutes of Health (NIH) National Institute of Environment Health Sciences [Grant R37 ES02710]; the National Institute of Environment Health Sciences Superfund Research Program [Grant P42 ES04699]; the National Institute of Diabetes and Digestive and Kidney Diseases [Grant R01 DK103616]; the NIH West Coast Comprehensive Metabolomics Resources Core [Grant U24 DK097154]; the National Institutes of Health Training Program [Grants T32 HL86350, T32 CA108459]; the Institute of General Medical Sciences Pharmacology Training Program [T32 GM099608]; and the American Association for Cancer research [Grant 16-4018-RAND].

https://doi.org/10.1124/mol.118.111831.

S This article has supplemental material available at molpharm. aspetjournals.org. ethanol or carbon tetrachloride (Liedtke et al., 2013). This inflammatory process involves activation of resident macrophages called hepatic stellate cells (HSCs) and the recruitment of macrophages, both of which express proinflammatory signaling molecules, along with enzymes and structural proteins that remodel the extracellular matrix (Pellicoro et al., 2014). This remodeling includes the increased deposition of matrix proteins such as collagen, as well as changes in the populations of metalloproteases (Pellicoro et al., 2014). If damage from exposure to the toxicant continues, liver fibrosis will alter the architecture of the organ and lead to liver failure (Liedtke et al., 2013).

Because of its anti-inflammatory effect, celecoxib has been explored as a possible therapy in several models of liver fibrosis, such as the thioacetamide (TAA)- and carbon tetrachloride $\left(\mathrm{CCl}_{4}\right)$-induced rodent models and the surgical bile duct ligation (BDL) rodent model. $\mathrm{CCl}_{4}$ acts primarily through an increase in hepatic lipid peroxidation and oxidative stress, whereas TAA acts primarily through an increase in oxidative stress, processes that damage hepatocytes and trigger fibrosis (Martínez et al., 2014). BDL is a surgical model in which the bile duct is partially ligated, leading to cholestasis, liver

ABBREVIATIONS: APAP, acetaminophen; BDL, bile duct ligation; $\mathrm{CCl}_{4}$, carbon tetrachloride; COX, cyclooxygenase; EET, epoxyeicosatrienoic acid; HSC, hepatic stellate cell; IHC, immunohistochemistry; LC-MS/MS, liquid chromatography-tandem mass spectrometry; LOX, lipoxygenase; $\mathrm{LXA}_{4}$, lipoxin A4; MMP, matrix metalloprotease; NSAID, nonsteroidal anti-inflammatory drug; $\mathrm{P} 450$, cytochrome $\mathrm{P} 450$; $P$ GD 2 , prostaglandin $\mathrm{D} 2$; $\mathrm{PGE}_{2}$, prostaglandin E2; $\mathrm{PGJ}_{2}$, prostaglandin J2; PTUPB, 4-(5-phenyl-3-\{3-[3-(4-trifluoromethylphenyl)-ureido]-propyl\}-pyrazol-1-yl)-benzenesulfonamide; sEH, soluble epoxide hydrolase; sEHI, soluble epoxide hydrolase inhibitor; SMA, smooth muscle actin; TAA, thioacetamide; TIMP, tissue inhibitor of metalloproteases; TGF, transforming growth factor; TPPU, 1-trifluoromethoxyphenyl-3-(1-propionylpiperidin-4-yl) urea. 
damage, and fibrosis (Martínez et al., 2014). In some models, treatment with celecoxib has resulted in a reduction in fibrosis and inflammation, whereas in others, including some rat $\mathrm{CCl}_{4}$-induced models, celecoxib has worsened liver damage and fibrosis (Hui et al., 2006; Paik et al., 2009; Chávez et al., 2010).

Celecoxib targets cyclooxygenase 2 (COX-2), an enzyme that metabolizes arachidonic acid to a class of oxidized fatty acids called prostaglandins (Shi and Klotz, 2008). These oxylipids have diverse effects in the liver, but many COX-2 metabolites increase inflammation and portal hypertension (Sacerdoti et al., 2015). Arachidonic acid is also metabolized by cytochrome P450s (P450s) to form the epoxyeicosatrienoic acids (EETs) (Morisseau and Hammock, 2013). The EETs have been investigated in several disease models and have been found to be anti-inflammatory, organ protective, and antifibrotic in heart and kidney models of fibrosis (Morisseau and Hammock, 2013). The EETs are further metabolized by soluble epoxide hydrolase $(\mathrm{sEH})$ to the dihydroxyeicosatrienoic acids that are less lipophilic and more readily conjugated and excreted by the organism (Morisseau and Hammock, 2013).

We previously modulated the oxylipids in a $\mathrm{CCl}_{4}$-induced model of hepatic fibrosis through dietary manipulation of lipid intake as well as inhibition of $\mathrm{sEH}$, which blocks the major route of metabolism of the EETs and other epoxy fatty acids (Harris et al., 2015, 2016). In general, perturbation of the oxylipids with sEH inhibitors reduced collagen deposition in addition to the expression and activity of profibrotic matrix metalloproteases (MMPs) (Harris et al., 2015, 2016). These results raised the question of how celecoxib, another modulator of oxylipids, would impact fibrosis in the $\mathrm{CCl}_{4}$ model and whether the tools we developed for inhibiting both $\mathrm{sEH}$ and COX-2 would alter the observed effects of COX-2 inhibition.

In this study, we treated mice with $\mathrm{CCl}_{4}$ over a 5 -week period to induce liver fibrosis. Interestingly, markers of fibrosis were elevated in the mice that received both celecoxib and $\mathrm{CCl}_{4}$ compared with those animals receiving $\mathrm{CCl}_{4}$ alone. Modulation of the EETs by either an $\mathrm{sEH}$ inhibitor or a dual inhibitor of COX-2 and sEH blunted some aspects of the profibrotic effect of COX-2 inhibition in the $\mathrm{CCl}_{4}$ background.

\section{Materials and Methods}

Animals. Male C57BL/6NCrl mice $(\sim 25 \mathrm{~g})$ were obtained from Charles River, Inc. (Wilmington, MA) 1 week before the experiment and kept on a 12-hour light/dark cycle. The animal protocol was approved by the University of California Davis Institutional Animal Care and Use Committee, and the study was performed in accordance with the National Institutes of Health guide for the care and use of laboratory animals.

Chemicals. All commercial chemicals were purchased from Sigma (St. Louis, MO) unless otherwise noted. TPPU, 1-(4-trifluoromethoxyphenyl)-3(1-propionylpiperidin-4-yl)-urea and PTUPB, 4-(5-phenyl-3-\{3-[3(4-trifluoromethylphenyl)-ureido]-propyl\}-pyrazol-1-yl)-benzenesulfonamide, were synthesized according to the previous procedures (Rose et al., 2010; Hwang et al., 2011). Celecoxib was a gift from Pfizer (New York, NY). Structures of TPPU, PTUPB, and celecoxib are given in Supplemental Fig. S1.

Experimental Design. The mice were randomly divided into five experimental groups (six animals/group): 1) control, 2) $\mathrm{CCl}_{4}$ only, 3) celecoxib $+\mathrm{CCl}_{4}, 4$ ) celecoxib $\left.+\mathrm{TPPU}+\mathrm{CCl}_{4}, 5\right) \mathrm{PTUPB}+\mathrm{CCl}_{4} \cdot \mathrm{CCl}_{4}$ was administered after a protocol described in detail in Constandinou et al. (2005). Briefly, $\mathrm{CCl}_{4}$ was mixed 1:1 (v/v) with Neobee M5 (Sigma) and injected i.p. every 5 days for a total of seven injections of $80 \mathrm{mg} / \mathrm{kg}$. Mice were euthanized 3 days after the final injection. Celecoxib, PTUPB, and a combination of celecoxib and TPPU were administered subcutaneously via Alzet model 2006 osmotic minipumps (Cupertino, $\mathrm{CA}$ ). These pumps produce a $3.6 \mu \mathrm{l} /$ day continuous flow rate for up to 45 days. The control group received Neobee M5 only. The control group and $\mathrm{CCl}_{4}$-only group were implanted with minipumps filled with the 1 : 1 (v/v) PEG400:DMSO solution.

Plasma Blood-Level Analysis. Mouse plasma (10 $\mu \mathrm{l})$ was mixed with $50 \mu \mathrm{l}$ of water containing $0.1 \%$ formic acid. The liquid-liquid extraction and LC-MS/MS analysis were performed as previously described (Harris et al., 2016).

Determination of Liver Tissue Hydroxyproline Levels. Hydroxyproline content was determined according to a method described in Reddy and Enwemeka (1996). Briefly, $10 \mu \mathrm{l}$ of $10 \mathrm{~N}$ $\mathrm{NaOH}$ was added to $40 \mu \mathrm{l}$ of $0.33 \mathrm{mg} / \mu \mathrm{l}$ liver tissue homogenate, and the solution was autoclaved at $120^{\circ} \mathrm{C}$ for 25 minutes. After the reaction cooled to room temperature, chloramine $\mathrm{T}$ reagent $(0.84 \%$ chloramine-T, $42 \mathrm{mM}$ sodium acetate, $2.6 \mathrm{mM}$ citric acid, and 39.5\% isopropanol) was added $(450 \mu \mathrm{l})$, bringing the volume to $500 \mu \mathrm{l}$. After incubation at room temperature for 25 minutes, DMAB reagent, $15 \%$ 4-(dimethylamino)benzaldehyde in 2:1 (v/v) isopropanol/perchloric acid mixture was added $(500 \mu \mathrm{l})$, bringing the volume to $1 \mathrm{ml}$. The solution was then incubated at $65^{\circ} \mathrm{C}$ for 20 minutes, and the absorbances of the samples measured at $550 \mathrm{~nm}$.

mRNA Transcript Analysis. Liver tissue was immediately stored in RNA later solution (Thermo Fisher Scientific, Waltham, MA) for 24 hours at $4^{\circ} \mathrm{C}$ before freezing at $-80^{\circ} \mathrm{C}$. Tissue was homogenized using a roto stator grinder (IKA Works Inc., Wilmington, NC) and passed through a QIAshredder column (Qiagen, Valencia, CA). Total RNA was purified using RNeasy kit (Qiagen). cDNA synthesis from total RNA was performed by the QuantiTect Reverse Transcription Kit (Qiagen). RT-PCR was performed using an Applied Biosciences Fast 7500 Real-Time PCR System (Foster City, CA). The following Taqman probes were purchased from Life Technologies Corporation: GAPDH, Mm99999915_g1; TGFB1, Mm01178820_m1; MMP-2, Mm00439498_m1; MMP-9, Mm00442991_m1; collagen 1A1, Mm00801666_g1; tissue inhibitors of metalloproteases (TIMP-1), Mm00441818_m1 (Life Technologies Corp., Grand Island, NY). GAPDH was used as the internal control.

Histopathology Scoring. Liver samples were immersion-fixed in $10 \%$ neutral-buffered formalin for 48 hours. Samples were stored in $70 \%(\mathrm{v} / \mathrm{v})$ ethanol in distilled deionized water before routine processing. Tissues were processed, embedded in paraffin, sectioned, and stained with H\&E by the histopathology service in the UC Davis Veterinary Medical Teaching Hospital (Davis, CA). H\&E-stained sections of liver were scored for inflammation, fibrosis, and cellular damage by a board-certified laboratory animal pathologist (D.M.I.). The scoring system is reported in Supplemental Table S1.

Trichrome Staining and Immunohistochemistry. $\alpha$ Smooth muscle actin (SMA) and F4/80 immunohistochemistry and trichrome staining were performed by the Genomic Pathology Laboratory at UC Davis and quantitated using imaging analysis software from Aperio (Sausalito, CA) using the IHC and positive pixel count algorithms.

Oxylipid Analysis. Oxylipids in the COX and lipoxygenase (LOX) branches of the arachidonic acid cascade were extracted and detected as described (Harris et al., 2016). Oxylipids in the P450 branch of the arachidonic acid cascade were detected as follows. Samples were analyzed on a Waters Acquity ultraperformance liquid chromatograph coupled to a Waters Xevo TQ-S Mass Spectrometer in negative electrospray ionization mode. Samples were injected $(5 \mu \mathrm{l})$ and separated using a Phenomenex Kinetex column $(150 \times 2.1 \mathrm{~mm} ; 1.7$ $\mu \mathrm{m})$ at $40^{\circ} \mathrm{C}$ using the following mobile-phase gradient, consisting of water (A) and acetonitrile (B), each containing $0.1 \%$ acetic acid: initial conditions of $65: 35 \mathrm{~A}: \mathrm{B}$ for 2.9 minutes ( $t=2.9$ minutes), changing to $45: 55$ at 3 minutes ( $t=3$ minutes) and decreasing to 35:65 over 5.5 minutes $(t=8.5)$, decreasing to $5: 95$ over 4 minutes $(t=12.5)$, holding at 5:95 for 1 minute $(t=13.5)$, reverting to initial conditions of 
65:35 in 0.1 minute $(t=13.6$ minutes) and re-equilibrating for 1.9 minutes ( $t=15.5$ minutes). Mass spectral analysis was accomplished using a capillary voltage of $3 \mathrm{kV}$, a desolvation temperature of $200^{\circ} \mathrm{C}$, a desolvation gas flow of 800 liters/hour, a cone gas flow of 150 liters/hour, nebulizer pressure of 6 bars, and collision gas flow of $0.15 \mathrm{ml} / \mathrm{min}$. Mass transitions for each EET were as follows: 14,15$\operatorname{EET}(319.0>219.0), 11,12-\mathrm{EET}(319.0>167.0)$, and 8,9-EET $(319.0>$ 155.0). Analyte concentration was quantified against an internal standard calibration curve, normalizing analyte response to 11,12 EET- $d_{11}(330.2>167.2)$, and 12-(3-cyclohexyl-ureido)dodecanoic acid $(341.3>216.4)$ to correct the extraction efficiency and instrument response, respectively. Dwell time for each analyte was 25 milliseconds. The detailed information for all analytes are reported in Supplemental Table S2.

Statistics. Normality was assessed by the Shapiro-Wilk test. Significance was determined by one-way analysis of variance, followed by the Dunnett multiple comparison test. All statistical calculations were performed using SigmaPlot (Systat Software, Inc., San Jose, CA).

\section{Results}

Subcutaneous Osmotic Minipumps Produced High Plasma Concentrations of Celecoxib. Male C57BL mice were implanted with osmotic minipumps 2 days before the start of $\mathrm{CCl}_{4}$ exposure via intraperitoneal injections as described in Materials and Methods. The five experimental groups were: 1) control, 2) $\mathrm{CCl}_{4}$ only, 3) celecoxib $\left.+\mathrm{CCl}_{4}, 4\right)$ celecoxib + TPPU $+\mathrm{CCl}_{4}$, and 5) PTUPB $+\mathrm{CCl}_{4}$. The drugs were loaded into minipumps so that the calculated dose of each compound would be $10 \mathrm{mg} / \mathrm{kg}$ per day.

When we examined plasma levels of the drugs, we found that the TPPU and celecoxib levels in the celecoxib + TPPU + $\mathrm{CCl}_{4}$ group were $1.11 \pm 0.018$ and $1.32 \pm 0.039 \mu \mathrm{M}$, respectively. The plasma concentration of celecoxib in the celecoxib $+\mathrm{CCl}_{4}$ group was $1.58 \pm 0.47 \mu \mathrm{M}$, and the PTUPB concentration in the PTUPB $+\mathrm{CCl}_{4}$ group was $0.65 \pm 0.027$ $\mu \mathrm{M}$. These plasma concentrations are far above the $\mathrm{IC}_{50}$ of for celecoxib and TPPU with their respective target mouse enzymes and approximately half the $\mathrm{IC}_{50}$ for PTUPB with human COX-2 and far above the $\mathrm{IC}_{50}$ for PTUPB with human $\mathrm{sEH}$. Despite developing robust fibrosis after the 5 -week $\mathrm{CCl}_{4}$ treatment, the weight of the mice increased, with no statistical difference between the groups $(P<0.05)$, the $\mathrm{CCl}_{4}$ toxicity being less severe than other models (Supplemental Fig. S2).

Celecoxib Increased Collagen Deposition and Liver Damage in $\mathbf{C C l}_{4}$-Treated Mice. Trichrome staining revealed that the celecoxib $+\mathrm{CCl}_{4}$ had a higher amount of collagen deposition than the $\mathrm{CCl}_{4}$-only group (Fig. 1, A-F). The PTUPB $+\mathrm{CCl}_{4}$ group had a reduced level of collagen deposition compared with the celecoxib $+\mathrm{CCl}_{4}$ group, although still higher than the control group (Fig. 1F). Hydroxyproline levels in hepatic tissue from the celecoxib $+\mathrm{CCl}_{4}$ group was the same as the $\mathrm{CCl}_{4}$-only group $(P>0.05)$, and the levels from both the celecoxib + TPPU $+\mathrm{CCl}_{4}$ and PTUPB + $\mathrm{CCl}_{4}$ groups were lower than the $\mathrm{CCl}_{4}$ only group (Fig. 1G).

We next performed a histopathological assessment of the extent of liver fibrosis and damage (Table 1). The categories were defined as follows: vacuolation-lacy or vacuolated cytoplasm that distends the hepatocyte; necrosis-single cell death of hepatocytes in centrilobular to random areas; lipofuscinosis-accumulation of lipid pigments, associated with nondegradable cellular breakdown products in macrophages; fibrosis-fibroblast proliferation with increased deposition of collagen around centrilobular regions occasionally connecting between centrilobular regions (bridges) or extending into the surrounding parenchyma (dissecting);
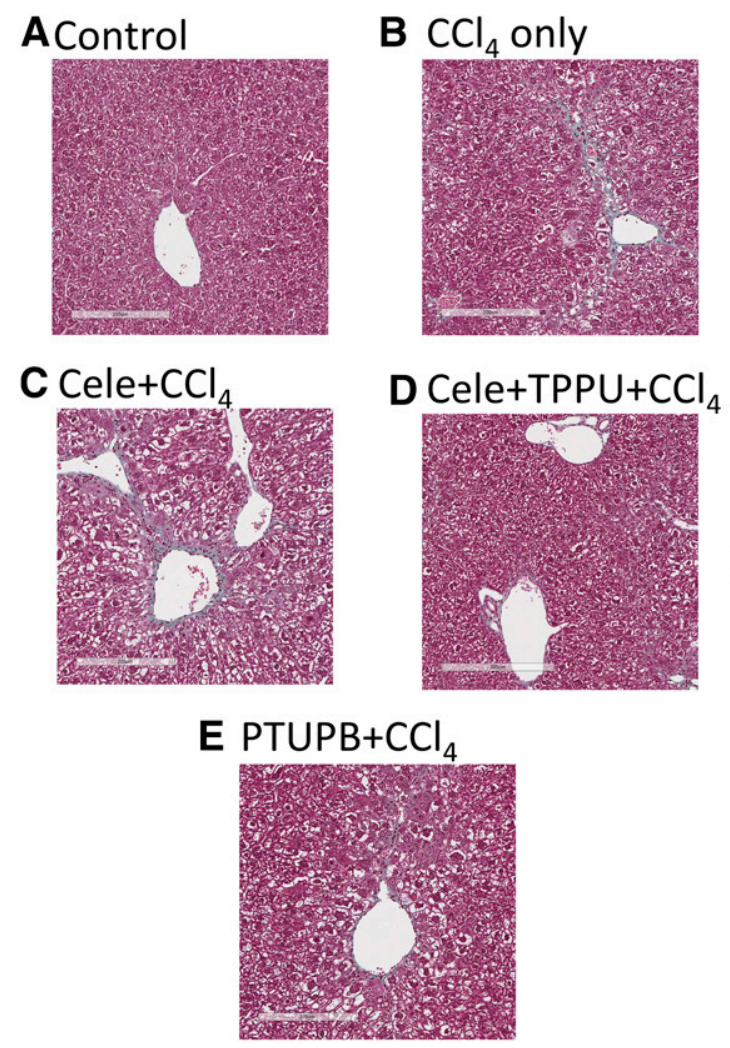

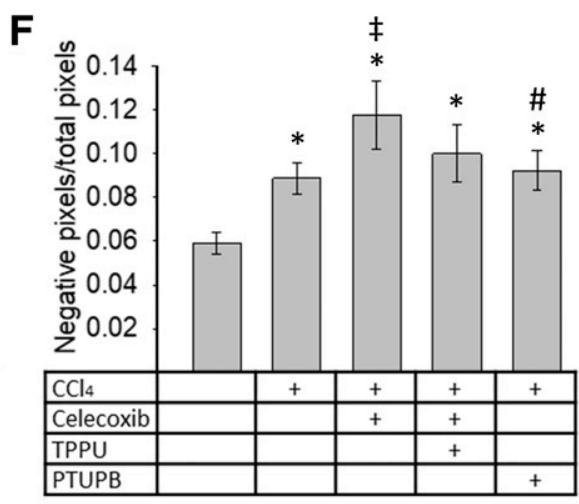

G

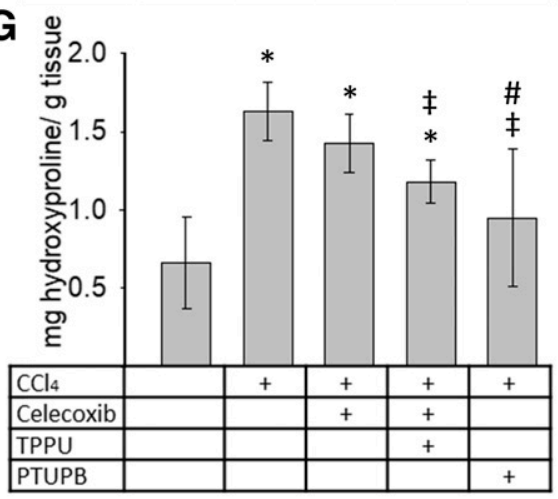

Fig. 1. Celecoxib increases the collagen deposition caused by $\mathrm{CCl}_{4}$ treatment. Mice were injected intraperitoneally with $\mathrm{CCl}_{4}$ for 5 weeks. The inhibitors were administered subcutaneously via osmotic minipumps that delivered the compounds at a calculated dose of $10 \mathrm{mg} / \mathrm{kg}$ per day for a 30-g mouse. (A-E) Representative slides of liver sections stained with trichrome (original magnification, 40×). (F) Quantification of staining as determined by the Aperio positive pixel count algorithm. The negative pixel ratio represents the percent area of collagen deposition, which is stained blue by the Trichrome stain. (G) Quantification of hepatic levels of hydroxyproline. Error bars represent S.D. $* P$ value vs. control group $<0.05 .{ }^{\ddagger} P$ value vs. $\mathrm{CCl}_{4}$-only group $<0.05$. ${ }^{\#} P$ value vs. celecoxib $+\mathrm{CCl}_{4}$ group $<0.05$. $n=6$ for celecoxib $+\mathrm{CCl}_{4}$ group, $n=5$ for all other groups. Statistical tests are described in Materials and Methods. The raw data used for this figure are reported in Supplemental Table S3. 
TABLE 1

Histologic scoring of H\&E-stained sections for inflammation, fibrosis and cellular damage on a 0-3 scale as described in Supplemental Table S1

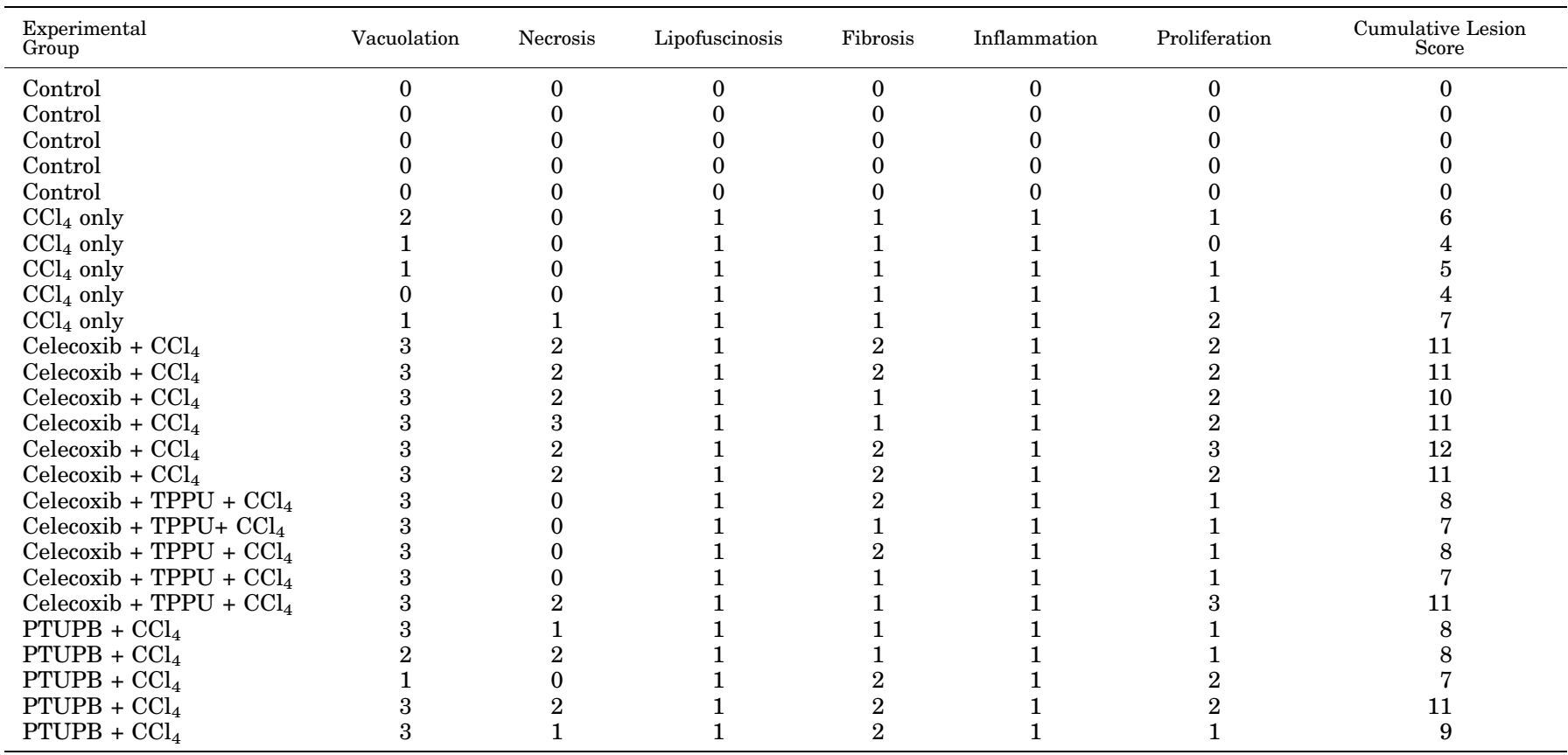

inflammation-mixed neutrophilic and mononuclear inflammation around predominantly centrilobular regions. The slides were read in a blind fashion. The scoring system is reported in Supplemental Table S1. The celecoxib $+\mathrm{CCl}_{4}$ group had the highest composite lesion scores owing to greater indices of hepatocellular damage (vacuolar degeneration, necrosis, fibrosis, regeneration). Examination of the celecoxib + TPPU +
$\mathrm{CCl}_{4}$ group showed that the addition of TPPU dampened the effect of celecoxib ( $P=0.0117)$, predominantly by decreasing hepatic necrosis $(P=0.0256)$ and regeneration $(P=0.0134)$.

Celecoxib Did Not Significantly Raise the Level of Profibrotic Markers in $\mathbf{C C l}_{4}$ - Treated Mice. To gauge further the extent of fibrosis, we measured the mRNA level of common markers by RTPCR (Fig. 2). The expression levels of
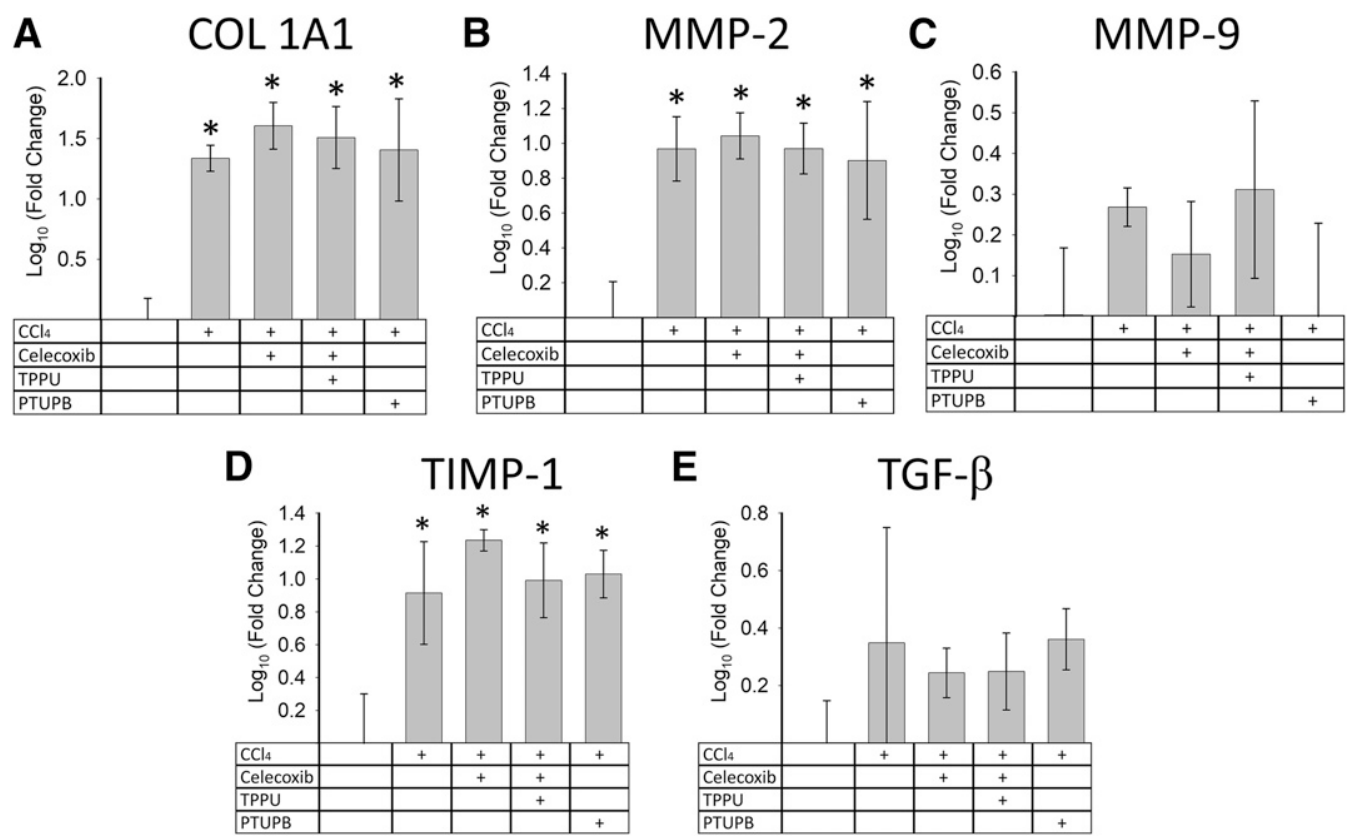

Fig. 2. Celecoxib modulates markers of hepatic fibrosis that are increased after $\mathrm{CCl}_{4}$ treatment. (A-E) mRNA expression of markers of fibrosis and inflammation. Total RNA from hepatic tissue and cDNA were prepared as described. Quantitative PCR was performed using Taqman probes on an Applied Biosystems Fast PCR instrument as described. Mice were injected intraperitoneally with $\mathrm{CCl}_{4}$ for 5 weeks. The inhibitors were administered subcutaneously via osmotic minipumps that delivered the compounds at a calculated dose of $10 \mathrm{mg} / \mathrm{kg}$ per day for a 30 -g mouse. Error bars represent S.D. $* P$ value vs. control group $<0.05 . n=3$ for celecoxib $+\mathrm{CCl}_{4}$ group, $n=4$ for all other groups. Statistical tests are described in Materials and Methods. The raw data used for this figure are reported in Supplemental Table S3. 
collagen $1 \mathrm{~A} 1$ (COL 1A1) was increased in the $\mathrm{CCl}_{4}$-only group compared with the control group $(P<0.05)$ and further increased in the celecoxib $+\mathrm{CCl}_{4}$ group, but this difference did not reach statistical significance $(P>0.05)$ (Fig. $2 \mathrm{~A})$. The mRNA expression of MMP-2 and MMP-9 were also elevated in the $\mathrm{CCl}_{4}$-only group (Fig. 2, B and C). For MMP-2, the three treatment groups were not different from the $\mathrm{CCl}_{4}$-only group (Fig. 2B). In the case of MMP-9, levels in the celecoxib $+\mathrm{CCl}_{4}$, celecoxib + TPPU $+\mathrm{CCl}_{4}$ and PTUPB $+\mathrm{CCl}_{4}$ groups did not differ from the $\mathrm{CCl}_{4}$-only group, but were also no different than the control group $(P>0.05)$ (Fig. 2C). TIMP-1 was elevated in the $\mathrm{CCl}_{4}$-only group, but the further increase observed in the celecoxib $+\mathrm{CCl}_{4}$, celecoxib $+\mathrm{TPPU}+\mathrm{CCl}_{4}$, and PTUPB $+\mathrm{CCl}_{4}$ groups did not reach significance (Fig. 2D). For TGF- $\beta$, the levels of the celecoxib $+\mathrm{CCl}_{4}$ and celecoxib + TPPU $+\mathrm{CCl}_{4}$ groups were lower than the $\mathrm{CCl}_{4^{-}}$ only group, but this difference did not reach statistical significance (Fig. 2E).

Celecoxib Increased $\alpha$ SMA But Not F4/80 Staining in $\mathbf{C C l}_{4}$-Treated Mice. $\mathrm{F} 4 / 80$ is a mouse cell surface protein expressed in many populations of macrophages. $\mathrm{CCl}_{4}$ increased the $\mathrm{F} 4 / 80$ immunohistochemical staining relative to the control group (Fig. 3). Whereas the amount of positive staining in the celecoxib $+\mathrm{CCl}_{4}$, PTUPB $+\mathrm{CCl}_{4}$, and celecoxib + TPPU $+\mathrm{CCl}_{4}$ groups were not significantly reduced $(P>0.05)$ compared with the $\mathrm{CCl}_{4}$-only group (Fig. $3 \mathrm{~F}$ ), these groups were also not different from the control group.

To assess hepatic stellate cell (HSC) activation, we performed IHC on liver tissue with an anti- $\alpha$ SMA (smooth muscle actin) antibody (Fig. 4). HSC activation was greatest in the celecoxib $+\mathrm{CCl}_{4}$ group, where it was significantly increased relative to both the control and $\mathrm{CCl}_{4}$-only groups $(P<0.05)$. Cotreatment with celecoxib and TPPU or treatment with
PTUPB in the $\mathrm{CCl}_{4}$ background decreased the level of HSC activation compared with animals receiving celecoxib and $\mathrm{CCl}_{4}$. In the case of the celecoxib $+\mathrm{TPPU}+\mathrm{CCl}_{4}$ group, HSC activation was significantly less than the celecoxib $+\mathrm{CCl}_{4}$ group $(P<0.05)$. In the PTUPB $+\mathrm{CCl}_{4}$ group, the level of HSC activation was not statistically different from the $\mathrm{CCl}_{4}$-only group $(P<0.05)$, unlike the celecoxib $+\mathrm{CCl}_{4}$ group.

Celecoxib Lowered the Tissue Level of COX Metabolites of Arachidonic Acid in $\mathbf{C C l}_{4}$-Treated Mice. We next examined the hepatic oxylipids by LC-MS/MS after solid-phase extraction of lipids from tissue lysates. A simplified diagram of these pathways is given in Supplemental Fig. S1. Although the differences did not reach statistical significance for every analyte, $\mathrm{CCl}_{4}$ treatment displayed the same trend across several COX metabolites, raising their concentration relative to the control group (Fig. 5; Supplemental Table S4). Three metabolites in the COX branch of the arachidonic acid cascade were significantly altered in the celecoxib $+\mathrm{CCl}_{4}$ group relative to either the control or the $\mathrm{CCl}_{4}$-only group (Fig. 5, A-D). The prostaglandin $\mathrm{E}_{2}$ and $\mathrm{PGD}_{2}$ levels were significantly higher in the $\mathrm{CCl}_{4}$-only group compared with the celecoxib $+\mathrm{CCl}_{4}$ group. Although they did not reach the level of statistical significance, prostaglandin $\mathrm{J}_{2}\left(\mathrm{PGJ}_{2}\right)$ and 15-deoxy- $\Delta^{12,14}-\mathrm{PGJ}_{2}$ showed similar trends, the celecoxib + $\mathrm{CCl}_{4}$ group being lower than the $\mathrm{CCl}_{4}$-only group. Interestingly, the LOX metabolite of arachidonic acid, lipoxin $\mathrm{A}_{4}\left(\mathrm{LXA}_{4}\right)$, was significantly elevated by celecoxib (Fig. 5E). The complete oxylipid profile is summarized in Supplemental Table S4.

Finally, we determined the effect of the treatments on hepatic EET levels (Supplemental Fig. S3; Supplemental Table S4). Although the differences in the levels of these analytes did not achieve statistical significance between any of the groups, the means for 8,9-, 11,12-, and 14,15-EET were elevated in $\mathrm{CCl}_{4}$-only group relative to the control group

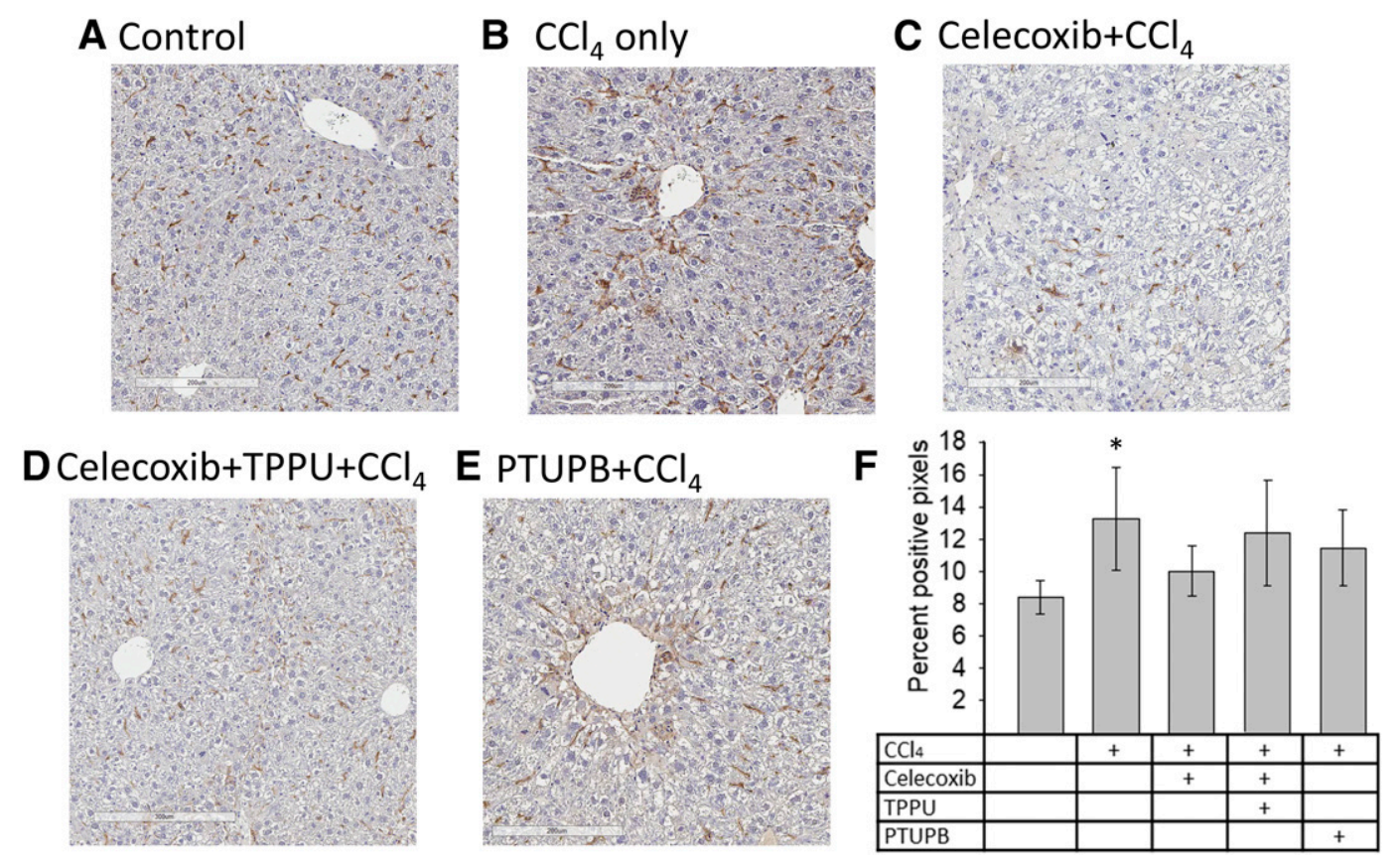

Fig. 3. Celecoxib attenuates the increased recruitment of macrophages caused by $\mathrm{CCl}_{4}$ treatment. (A-E) Immunohistochemistry (IHC) on liver tissue with an anti-F4/80 antibody (original magnification, $40 \times$ ). (F) Quantification of IHC stain using the Aperio positive pixel count algorithm. Mice were injected intraperitoneally with $\mathrm{CCl}_{4}$ for 5 weeks. The inhibitors were administered subcutaneously via osmotic minipumps that delivered the compounds at a calculated dose of $10 \mathrm{mg} / \mathrm{kg}$ per day for a 30 -g mouse. Error bars represent S.D. $* P$-value vs. control group $<0.05$. $n=5$. Statistical tests are described in Materials and Methods. The raw data used for this figure are reported in Supplemental Table S3. 


\section{A Control}

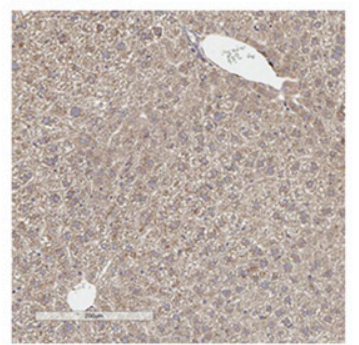

DCelecoxib+TPPU+CCl ${ }_{4} \mathrm{E}$

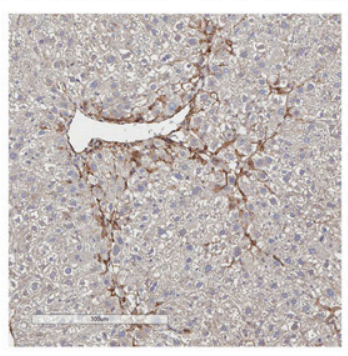

B $\mathrm{CCl}_{4}$ only
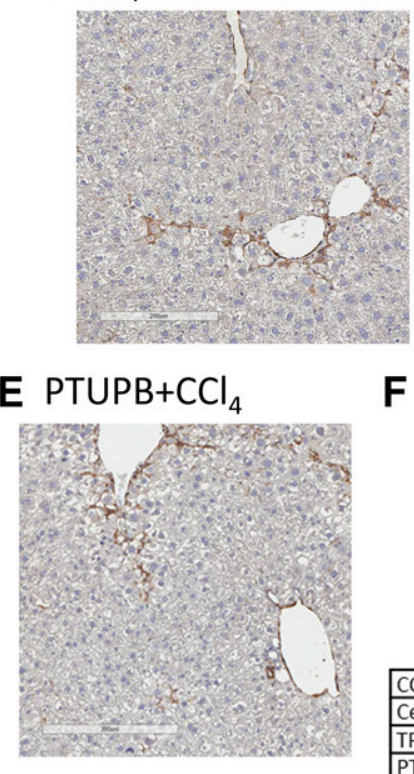

\section{Celecoxib $+\mathrm{CCl}_{4}$}

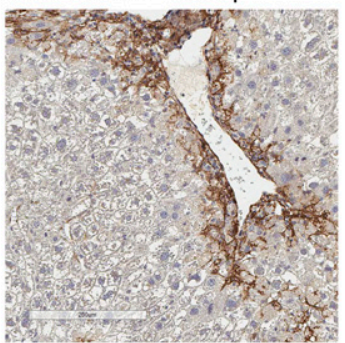

\section{$\mathbf{F}$}

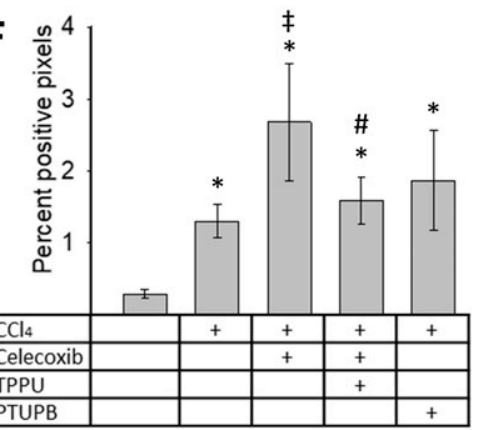

Fig. 4. Celecoxib further increases the hepatic stellate cell activation triggered by $\mathrm{CCl}_{4}$ treatment. (A-E) Immunohistochemistry on liver tissue with an anti$\alpha$ SMA antibody (original magnification, $40 \times$ ). (F) Quantification of stain using the Aperio positive pixel count algorithm. Mice were injected (intraperitoneally) with $\mathrm{CCl}_{4}$ for 5 weeks. The inhibitors were administered subcutaneously via osmotic minipumps that delivered the compounds at a calculated dose of $10 \mathrm{mg} / \mathrm{kg}$ per day for a $30-\mathrm{g}$ mouse. Error bars represent S.D. $* P$ value vs. control group $<0.05$. ${ }^{\ddagger} P$ value vs. $\mathrm{CCl}_{4}$ only group $<0.05$. ${ }^{\#} P$ value vs. celecoxib $+\mathrm{CCl}_{4}$ group $<0.05 . n=5$. Statistical tests are described in Materials and Methods. The raw data used for this figure are reported in Supplemental Table S3.
(Supplemental Fig. S3, A-C). The celecoxib $+\mathrm{CCl}_{4}$ group had lowered 11,12- and 14,15- levels relative to the control group (Supplemental Fig. S3, B and C). In the celecoxib + TPPU + $\mathrm{CCl}_{4}$ and $\mathrm{PTUPB}+\mathrm{CCl}_{4}$ groups, the EET levels were increased relative to the celecoxib $+\mathrm{CCl}_{4}$ group (Supplemental Fig. S3, $\mathrm{A}-\mathrm{C})$.

\section{Discussion}

There is a current disagreement in the literature regarding the use of celecoxib as a treatment of liver damage and fibrosis (Hui et al., 2004; Paik et al., 2009; Yu et al., 2009; Wen et al., 2014). Relevant to this study, opposing results have been reported in $\mathrm{CCl}_{4}$ models of liver fibrosis. In a short-term model, celecoxib reduced liver inflammation and damage during the early phase of $\mathrm{CCl}_{4}$ toxicity (Washino et al., 2010). A similar short-term study found that celecoxib reduced markers of lipid peroxidation but had no effect on hepatic toxicity or necrosis (Ekor et al., 2013). Chávez et al. (2010) reported that celecoxib reduced fibrosis as judged by collagen deposition and other biochemical markers; however, Hui et al. (2006) reported that treatment with celecoxib increased hepatic fibrosis, gauged by collagen deposition and MMP production. In their discussion of these $\mathrm{CCl}_{4}$ studies, Chávez et al. (2010) suggested that differences in $\mathrm{CCl}_{4}$ model parameters might explain the conflicting results, in particular, the dose and length of time of toxicant exposure.
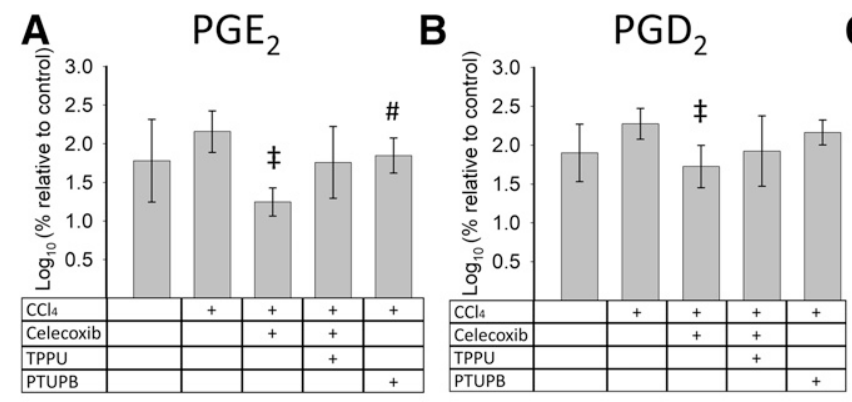

D 15 -deoxy- $\Delta 12,14-$ PGJ $_{2}$

E

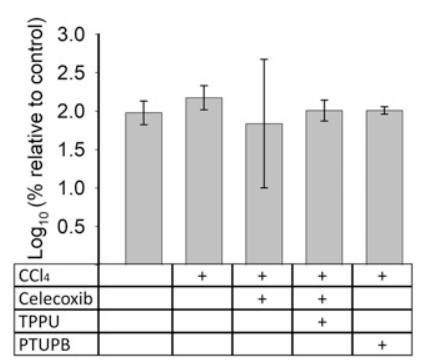

C

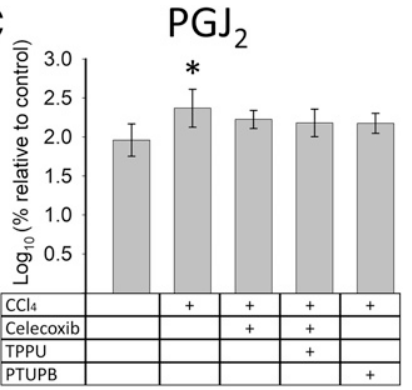

Lipoxin $\mathrm{A}_{4}$

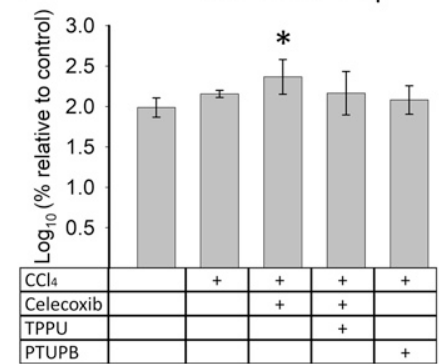

Fig. 5. Celecoxib modulates the increase in metabolites in the COX and LOX branches of the arachidonic acid cascade caused by $\mathrm{CCl}_{4}$ treatment. (A-D) Hepatic tissue levels of metabolites in the COX branch. (E) Hepatic tissue levels of lipoxin $\mathrm{A}_{4}$, a metabolite in the LOX branch. Tissue levels of compounds were analyzed by LC-MS/MS after solid-phase extraction as described in Materials and Methods. Mice were injected (intraperitoneally) with $\mathrm{CCl}_{4}$ for 5 weeks. The inhibitors were administered subcutaneously via osmotic minipumps that delivered the compounds at a calculated dose of $10 \mathrm{mg} / \mathrm{kg}$ per day for a $30 \mathrm{-g}$ mouse. For a simplified diagram of these pathways, see Supplemental Fig. S1. Error bars represent S.D. $* P$ value vs. control group $<0.05$. ${ }^{\ddagger} P$ value vs. $\mathrm{CCl}_{4}$ only group $<0.05$. ${ }^{\sharp} P$ value vs. celecoxib $+\mathrm{CCl}_{4}$ group $<0.05 . n=6$ for celecoxib $+\mathrm{CCl}_{4}$ group, $n=5$ for all other groups. Statistical tests are described in Materials and Methods. The raw data used for this figure are reported in Supplemental Table S3. 
In the current study, we obtained results similar to those reported by Hui et al. (2006) after treatment with celecoxib in their $\mathrm{CCl}_{4}$ rat model of liver fibrosis, as well as those obtained by Reilly et al. (2001) using COX-2 knockout mice in an acetaminophen (APAP) model of acute liver damage. Like Hui et al. (2006), we found that celecoxib treatment increased the amount of collagen deposition by approximately $25 \%$ compared with the $\mathrm{CCl}_{4}$-only group. We also observed an increase in $\alpha$ SMA staining, a marker for activated HSCs, in the celecoxib $+\mathrm{CCl}_{4}$ group compared with the $\mathrm{CCl}_{4}$-only group, although our model produced an almost 2-fold increase compared with their reported $40 \%$ increase (Hui et al., 2006); however, we did not see an increase in MMP-2 or MMP-9 mRNA expression in the celecoxib $+\mathrm{CCl}_{4}$ group compared with the $\mathrm{CCl}_{4}$-only group, unlike Hui and colleagues. It should be kept in mind that Hui et al. treated with a much higher dose of $\mathrm{CCl}_{4}$ (i.e., $3200 \mathrm{mg} / \mathrm{kg}$ delivered twice weekly) compared with our $80 \mathrm{mg} / \mathrm{kg}$ delivered twice weekly. Our study also parallels results using COX-2 transgenic mice in a liver damage model. When COX-2-deficient mice were given a single bolus of APAP in an acute liver damage model (Reilly et al., 2001), the $\mathrm{COX}^{-/-}$and $\mathrm{COX}^{-/+}$animals displayed greater bridging perivenous hepatocyte necrosis than did wildtype (Reilly et al., 2001). Similarly, we observed an increase in hepatic necrosis in the celecoxib $+\mathrm{CCl}_{4}$ group compared with the $\mathrm{CCl}_{4}$-only group. Although the mechanism of toxicity of APAP and $\mathrm{CCl}_{4}$ are different, this may indicate that celecoxib accelerates hepatic necrosis in models that involve severe oxidative stress.

We used two pharmacologic tools in addition to celecoxib to perturb the oxylipid homeostasis, TPPU and PTUPB. TPPU is a potent inhibitor of soluble epoxide hydrolase, an enzyme that metabolizes epoxy fatty acids (Rose et al., 2010). In previous studies, we have reported that TPPU treatment reduces hepatic fibrosis (Harris et al., 2015, 2016). PTUPB contains the pharmacophores from both celecoxib and TPPU and inhibits both enzymes (Hwang et al., 2011). Treatment with PTUPB or cotreatment with TPPU and celecoxib decreased the elevated collagen deposition observed in the celecoxib $+\mathrm{CCl}_{4}$ group. The most striking result obtained with these inhibitors concerns the elevated $\alpha$ SMA in the celecoxib $+\mathrm{CCl}_{4}$ group. Although not returning $\alpha \mathrm{SMA}$ levels to that of the control group, cotreatment with the $\mathrm{sEH}$ inhibitor attenuated the elevated $\alpha$ SMA expression observed in the celecoxib $+\mathrm{CCl}_{4}$ group. Changes in associated oxylipid mediators are discussed subsequently herein. We saw a slight reduction in $\mathrm{F} 4 / 80$ expression, a marker of macrophages, in the celecoxib $+\mathrm{CCl}_{4}$ group compared with the $\mathrm{CCl}_{4}$-only group, indicating that the increase in fibrosis in the celecoxib $+\mathrm{CCl}_{4}$ group is not due to increased macrophage migration.

The downstream COX-2 metabolites prostaglandin E2 $\left(\mathrm{PGE}_{2}\right)$ and 15-deoxy- $\Delta^{12,14}-\mathrm{PGJ}_{2}$ have been proposed to mediate both the profibrotic and antifibrotic effects of COX-2 inhibition or gene deletion, depending on the model. In general, prostaglandins have organ-protective effects in the liver (Reilly et al., 2001), and $\mathrm{PGE}_{2}$ reduced collagen production in a human cell culture model of HSCs (Hui et al., 2004). Results that support a profibrotic role for COX-2 downstream metabolites include the observation that plasma $\mathrm{PGE}_{2}$ levels were elevated after treatment with TAA (Wen et al., 2014). In a separate study using the BDL and TAA models, $\mathrm{PGE}_{2}$ levels were increased in the fibrotic animals and reduced after treatment with celecoxib, with an accompanying attenuation of fibrosis (Paik et al., 2009). 15-deoxy- $\Delta^{12,14}-$ PGJ $_{2}$ is another potential profibrotic downstream COX-2 metabolite elevated in the serum of fibrotic rats treated with $\mathrm{CCl}_{4}$ (Planagumà et al., 2005); however, 15-deoxy- $\Delta^{12,14}-\mathrm{PGJ}_{2}$ has also been shown to induce apoptosis of human myofibroblasts, a process that contributes to the resolution of fibrosis ( $\mathrm{Li}$ et al., 2001).

We performed an analysis of oxylipids of the arachidonic acid cascade to determine the effect of COX-2 and sEH inhibition. Overall, COX metabolites were elevated in the $\mathrm{CCl}_{4}$-only group, although the levels did not reach statistical significance in most cases. $\mathrm{PGE}_{2}$ levels were elevated in the $\mathrm{CCl}_{4}$-only group and substantially lowered in the celecoxib + $\mathrm{CCl}_{4}$ group, as expected for a COX inhibitor. As outlined before, extensive fibrosis was seen in both these groups; the greatest degree of fibrosis was found in the celecoxib $+\mathrm{CCl}_{4}$ group, where the lowest $\mathrm{PGE}_{2}$ level was detected. Consistent with an anti-inflammatory effect of celecoxib in the liver, $\mathrm{LXA}_{4}$ was greatly elevated by celecoxib. Treatment with TPPU and celecoxib or the dual COX-2/sEH inhibitor in the $\mathrm{CCl}_{4}$ background raised $\mathrm{PGE}_{2}$ levels and lowed $\mathrm{LXA}_{4}$ levels compared with the celecoxib $+\mathrm{CCl}_{4}$ group, in both cases bringing the levels of these lipid mediators closer to those observed in the control group. It is possible that a drastic reduction in inflammatory processes in the $\mathrm{CCl}_{4}$ background results in even greater damage to the liver and that, depending on the inflammatory state of the liver, an elevation in $\mathrm{PGE}_{2}$ levels will either increase or decrease liver damage and fibrosis, which might explain some of the contradictory conclusions regarding the role of $\mathrm{PGE}_{2}$ in different liver fibrosis models.

Wide variation was seen in the $\mathrm{PGJ}_{2}$ and 15-deoxy- $\Delta^{12,14}$ $\mathrm{PGJ}_{2}$ levels, but the trend of the metabolites was similar. Whereas $\mathrm{CCl}_{4}$ elevated their mean levels compared with the control group, levels were slightly decreased in other groups. Based on these data, the 15-deoxy- $\Delta^{12,14}-\mathrm{PGJ}_{2}$ levels do not explain the increased fibrosis in the celecoxib $+\mathrm{CCl}_{4}$ group or the attenuation in fibrosis after TPPU cotreatment.

sEH inhibitors, and presumably the underlying elevation in epoxy fatty acid chemical mediators, have reduced pathologic fibrosis in several models (Kim et al., 2014; Hye Khan et al., 2016; Liao et al., 2016; Zhou et al., 2016), including ischemiadriven heart failure (Sirish et al., 2013). Earlier we demonstrated that soluble epoxide hydrolase inhibitor (sEHI) reduced hepatic fibrosis in the same $\mathrm{CCl}_{4}$ model used in this study (Harris et al., 2015, 2016); however, unlike some other pathologic changes reversed by sEHI, the effects were not enhanced by a diet depleted in $\omega-6$ relative to $\omega-3$ fatty acids.

sEH inhibitors synergize with NSAIDs in many biologic systems (Schmelzer et al., 2006; Hwang et al., 2011; Zhang et al., 2014; Hye Khan et al., 2016). Surprisingly, sEH inhibitors prevent and reverse gastrointestinal erosion caused by the NSAIDs diclofenac (Goswami et al., 2016, 2017), and indomethacin (Goswami, unpublished), and in mice they block some of the cardiovascular effects caused by COX-2 inhibitors, possibly by returning the enhanced blood levels of thromboxane A2 to prostaglandin I2 to more normal levels (Schmelzer et al., 2006). Since celecoxib is a selective COX-2 inhibitor and PTUPB is a highly selective COX-2 inhibitor, we expected a strong positive synergistic effect between the sEHI TPPU and celecoxib and a dramatic reduction in fibrosis with PTUPB. 
Surprisingly, the profibrotic effect of celecoxib in our $\mathrm{CCl}_{4}$ model overpowered possible positive synergistic interaction with sEHI, giving only moderate efficacy in reducing fibrosis and fibrosis markers. This result suggests that celecoxib in not an attractive drug to treat hepatic fibrosis in a system driven by pathologic peroxidation; however, both sEHI alone and dual COX/sEH inhibitors may be worth examining in hepatic fibrosis models driven by agents other than $\mathrm{CCl}_{4}$. In patients with hepatic fibrosis who are on celecoxib or other NSAIDs for a different disease, sEHI may ameliorate exacerbation of hepatic fibrosis by celecoxib.

Finally, although differences observed in the EETs did not achieve statistical significance, the trends suggest that the sEH inhibitors were able to elevate hepatic EET levels, although $\mathrm{CCl}_{4}$ treatment also raised the level of these oxylipids. Given the variation in the data, one must use caution in interpretation, but it is possible that the slight increase in EET levels after $\mathrm{CCl}_{4}$ treatment is insufficient to counteract a profibrotic environment and that the further increase in EETs caused by sEH inhibition tips the balance, partially countering the profibrotic effects of celecoxib in the $\mathrm{CCl}_{4}$ background. In the oxylipin, as well as the mRNA expression and histologic analyses, small sample sizes might be responsible for trends not reaching significance in this study. Given the variability of fibrotic and inflammatory responses in chronic studies, small group sizes can limit the ability to detect differences between groups.

Because of the differences in animal models and the dose of celecoxib used, the effectiveness of COX-2 inhibition as a treatment of liver fibrosis is questionable. In the current study, we have an indication that oxidized fatty acids other than COX-2 metabolites of arachidonic acid may be involved in the profibrotic properties of celecoxib in a $\mathrm{CCl}_{4}$ mouse model of liver fibrosis. Given their effectiveness in modulating the EETs, as well as their antifibrotic effects in the liver and other tissues, sEHIs are promising tools for the study of the role of these oxylipids in hepatic fibrosis and damage.

\section{Authorship Contributions}

Participated in research design: Harris, Kodani, Hammock.

Conducted experiments: Harris, Rand.

Contributed new reagents or analytic tools: Rand, Yang, Hwang.

Performed data analysis: Harris, Imai.

Wrote or contributed to the writing of the manuscript: Harris, Imai, Hammock.

\section{References}

Bessone F, Hernandez N, Roma MG, Ridruejo E, Mendizabal M, Medina-Cáliz I, Robles-Díaz M, Lucena MI, and Andrade RJ (2016) Hepatotoxicity induced by coxibs: how concerned should we be? Expert Opin Drug Saf 15:1463-1475.

Chávez E, Segovia J, Shibayama M, Tsutsumi V, Vergara P, Castro-Sánchez L, Salazar EP, Moreno MG, and Muriel P (2010) Antifibrotic and fibrolytic properties of celecoxib in liver damage induced by carbon tetrachloride in the rat. Liver Int 30:969-978.

Constandinou C, Henderson N, and Iredale JP (2005) Modeling liver fibrosis in rodents. Methods Mol Med 117:237-250.

Ekor M, Odewabi AO, Kale OE, Adesanoye OA, and Bamidele TO (2013) Celecoxib, a selective cyclooxygenase- 2 inhibitor, lowers plasma cholesterol and attenuates hepatic lipid peroxidation during carbon-tetrachloride-associated hepatotoxicity in rats. Drug Chem Toxicol 36:1-8.

Goswami SK, Rand AA, Wan D, Yang J, Inceoglu B, Thomas M, Morisseau C, Yang GY, and Hammock BD (2017) Pharmacological inhibition of soluble epoxide hydrolase or genetic deletion reduces diclofenac-induced gastric ulcers. Life Sci 180:114-122.

Goswami SK, Wan D, Yang J, Trindade da Silva CA, Morisseau C, Kodani SD, Yang GY, Inceoglu B, and Hammock BD (2016) Anti-ulcer efficacy of soluble epoxide hydrolase inhibitor TPPU on diclofenac-induced intestinal ulcers. J Pharmacol Exp Ther 357:529-536.

Harris TR, Bettaieb A, Kodani S, Dong H, Myers R, Chiamvimonvat N, Haj FG, and Hammock BD (2015) Inhibition of soluble epoxide hydrolase attenuates hepatic fibrosis and endoplasmic reticulum stress induced by carbon tetrachloride in mice. Toxicol Appl Pharmacol 286:102-111.
Harris TR, Kodani S, Yang J, Imai DM, and Hammock BD (2016) An $\omega$-3-enriched diet alone does not attenuate $\mathrm{CCl}_{4}$-induced hepatic fibrosis. $J$ Nutr Biochem 38:93-101. Hui AY, Dannenberg AJ, Sung JJ, Subbaramaiah K, Du B, Olinga P, and Friedman SL (2004) Prostaglandin E2 inhibits transforming growth factor beta 1-mediated induction of collagen alpha 1(I) in hepatic stellate cells. J Hepatol 41:251-258.

Hui AY, Leung WK, Chan HL, Chan FK, Go MY, Chan KK, Tang BD, Chu ES, and Sung JJ (2006) Effect of celecoxib on experimental liver fibrosis in rat. Liver Int 26:125-136.

Hwang SH, Wagner KM, Morisseau C, Liu JY, Dong H, Wecksler AT, and Hammock BD (2011) Synthesis and structure-activity relationship studies of urea-containing pyrazoles as dual inhibitors of cyclooxygenase- 2 and soluble epoxide hydrolase. $J$ Med Chem 54:3037-3050

Hye Khan MA, Hwang SH, Sharma A, Corbett JA, Hammock BD, and Imig JD (2016) A dual COX-2/sEH inhibitor improves the metabolic profile and reduces kidney injury in Zucker diabetic fatty rat. Prostaglandins Other Lipid Mediat 125:40-47.

Kim J, Imig JD, Yang J, Hammock BD, and Padanilam BJ (2014) Inhibition of soluble epoxide hydrolase prevents renal interstitial fibrosis and inflammation. Am $J$ Physiol Renal Physiol 307:F971-F980.

Li L, Tao J, Davaille J, Feral C, Mallat A, Rieusset J, Vidal H, and Lotersztajn S (2001) 15-deoxy-delta 12,14-prostaglandin J2 induces apoptosis of human hepatic myofibroblasts: a pathway involving oxidative stress independently of peroxisomeproliferator-activated receptors. J Biol Chem 276:38152-38158.

Liao J, Hwang SH, Li H, Liu JY, Hammock BD, and Yang GY (2016) Inhibition of chronic pancreatitis and murine pancreatic intraepithelial neoplasia by a dual inhibitor of c-RAF and soluble epoxide hydrolase in LSL-Kras ${ }^{\mathrm{G} 12 \mathrm{D}} / \mathrm{Pdx}-1-\mathrm{Cre}$ mice. Anticancer Res 36:27-37.

Liedtke C, Luedde T, Sauerbruch T, Scholten D, Streetz K, Tacke F, Tolba R, Trautwein C, Trebicka J, and Weiskirchen R (2013) Experimental liver fibrosis research: update on animal models, legal issues and translational aspects. Fibrogenesis Tissue Repair 6:19.

Martínez AK, Maroni L, Marzioni M, Ahmed ST, Milad M, Ray D, Alpini G, and Glaser SS (2014) Mouse models of liver fibrosis mimic human liver fibrosis of different etiologies. Curr Pathobiol Rep 2:143-153.

Morisseau C and Hammock BD (2013) Impact of soluble epoxide hydrolase and epoxyeicosanoids on human health. Annu Rev Pharmacol Toxicol 53:37-58.

Paik YH, Kim JK, Lee JI, Kang SH, Kim DY, An SH, Lee SJ, Lee DK, Han KH, Chon CY, et al. (2009) Celecoxib induces hepatic stellate cell apoptosis through inhibition of Akt activation and suppresses hepatic fibrosis in rats. Gut 58:1517-1527.

Pellicoro A, Ramachandran P, Iredale JP, and Fallowfield JA (2014) Liver fibrosis and repair: immune regulation of wound healing in a solid organ. Nat Rev Immunol 14:181-194.

Planagumà A, Clària J, Miquel R, López-Parra M, Titos E, Masferrer JL, Arroyo V, and Rodés J (2005) The selective cyclooxygenase-2 inhibitor SC-236 reduces liver fibrosis by mechanisms involving non-parenchymal cell apoptosis and PPARgamma activation. FASEB J 19:1120-1122.

Reddy GK and Enwemeka CS (1996) A simplified method for the analysis of hydroxyproline in biological tissues. Clin Biochem 29:225-229.

Reilly TP, Brady JN, Marchick MR, Bourdi M, George JW, Radonovich MF, PiseMasison CA, and Pohl LR (2001) A protective role for cyclooxygenase-2 in druginduced liver injury in mice. Chem Res Toxicol 14:1620-1628.

Rose TE, Morisseau C, Liu JY, Inceoglu B, Jones PD, Sanborn JR, and Hammock BD (2010) 1-Aryl-3-(1-acylpiperidin-4-yl)urea inhibitors of human and murine soluble epoxide hydrolase: structure-activity relationships, pharmacokinetics, and reduction of inflammatory pain. J Med Chem 53:7067-7075.

Sacerdoti D, Pesce P, Di Pascoli M, Brocco S, Cecchetto L, and Bolognesi M (2015) Arachidonic acid metabolites and endothelial dysfunction of portal hypertension. Prostaglandins Other Lipid Mediat 120:80-90.

Schmelzer KR, Inceoglu B, Kubala L, Kim IH, Jinks SL, Eiserich JP, and Hammock BD (2006) Enhancement of antinociception by coadministration of nonsteroidal anti-inflammatory drugs and soluble epoxide hydrolase inhibitors. Proc Natl Acad Sci USA 103:13646-13651.

Shi S and Klotz U (2008) Clinical use and pharmacological properties of selective COX-2 inhibitors. Eur J Clin Pharmacol 64:233-252.

Sirish P, Li N, Liu JY, Lee KS, Hwang SH, Qiu H, Zhao C, Ma SM, López JE, Hammock BD, et al. (2013) Unique mechanistic insights into the beneficial effects of soluble epoxide hydrolase inhibitors in the prevention of cardiac fibrosis. Proc Natl Acad Sci USA 110:5618-5623.

Washino Y, Koga E, Kitamura Y, Kamikawa C, Kobayashi K, Nakagawa T, Nakazaki C, Ichi I, and Kojo S (2010) Effect of celecoxib, a selective cyclooxygenase-2 inhibitor on carbon tetrachloride intoxication in rats. Biol Pharm Bull 33:707-709.

Wen SL, Gao JH, Yang WJ, Lu YY, Tong H, Huang ZY, Liu ZX, and Tang CW (2014) Celecoxib attenuates hepatic cirrhosis through inhibition of epithelial-tomesenchymal transition of hepatocytes. J Gastroenterol Hepatol 29:1932-1942.

Yu J, Hui AY, Chu ES, Go MY, Cheung KF, Wu CW, Chan HL, and Sung JJ (2009) The anti-inflammatory effect of celecoxib does not prevent liver fibrosis in bile ductligated rats. Liver Int 29:25-36.

Zhang G, Panigrahy D, Hwang SH, Yang J, Mahakian LM, Wettersten HI, Liu JY, Wang Y, Ingham ES, Tam S, et al. (2014) Dual inhibition of cyclooxygenase-2 and soluble epoxide hydrolase synergistically suppresses primary tumor growth and metastasis. Proc Natl Acad Sci USA 111:11127-11132.

Zhou Y, Yang J, Sun GY, Liu T, Duan JX, Zhou HF, Lee KS, Hammock BD, Fang X, Jiang JX, et al. (2016) Soluble epoxide hydrolase inhibitor 1-trifluoromethoxyphenyl-3- (1-propionylpiperidin-4-yl) urea attenuates bleomycin-induced pulmonary fibrosis in mice. Cell Tissue Res 363:399-409.

Address correspondence to: Bruce D. Hammock, Department of Entomology and Nematology and UC Davis Comprehensive Cancer Center, University of California, Davis, 1 Shields Avenue, Davis, CA 95616. E-mail: bdhammock@ ucdavis.edu 